\title{
A 24-year-old woman with episodes of shortness of breath, cough and wheezing
}

\author{
Vivek Dhawan MD PhD, Meena Kalluri MD
}

A 24-year-old woman presented to an emergency department with a 1-week history of increasing shortness of breath accompanied with productive cough and wheezing. These symptoms were preceded by those of an upper respiratory tract infection. She had been admitted to hospital recently for a similar presentation and had been discharged home with a diagnosis of asthma and prescribed bronchodilators, an inhaled corticosteroid, supplemental oxygen and a tapering dose of prednisone.

The patient was a current smoker with a 10 pack-year history of smoking. Her medical history included hepatitis $\mathrm{C}$, inhaled cocaine abuse, which she quit a year ago, a mood disorder and a learning disability. She had no history of atopy, sinus disease or gastroesophageal reflux and no family history of asthma. She lived independently with assistance from a local program for people with disabilities.

Over the previous year, she had visited her family physician, walk-in clinics and emergency departments multiple times because of episodes of dyspnea and wheezing, which were becoming more frequent. Her Medical Research Council dyspnea score ranged from $2 / 5$ on good days to $4 / 5$ on bad days. Her list of medications included a combined inhaled corticosteroid and longacting $\beta$-agonist, salbutamol, ipratropium bromide, dextroamphetamine sulfate, risperidone, sertraline hydrochloride, and vitamin D and calcium supplements.

In the emergency department, the patient required $7 \mathrm{~L}$ of supplemental oxygen by face mask. Decreased air entry and wheezes were heard bilaterally on auscultation of her lungs. No signs of congestive heart failure were noted; however, a loud pulmonic second sound $\left(\mathrm{P}_{2}\right)$ was heard on auscultation. The patient had an elevated body mass index of 33. Arterial blood gas levels on the supplemental oxygen were consistent with mixed respiratory failure: partial pressure of arterial oxygen $75 \mathrm{~mm} \mathrm{Hg}$, and partial pressure of arterial carbon dioxide $51 \mathrm{~mm} \mathrm{Hg}$. A chest radio- graph showed minor atelectasis in the mid-lower zone of the left lung, with no signs of hyperinflation or consolidation. A working diagnosis of difficult-to-treat asthma was made, and treatment with bronchodilators and systemic steroid and antibiotic therapy was started.

On review of the patient's previous investigations, we noted that when she was relatively well, results of pulmonary function testing showed severe fixed airflow obstruction with air trapping (a ratio of forced expiratory volume in the first second of expiration to forced vital capacity $\left[\mathrm{FEV}_{1} / \mathrm{FVC}\right]$ of 0.54 and an $\mathrm{FEV}_{1}$ of $1.50 \mathrm{~L}$ [43\% predicted], with no substantial change after bronchodilator use). The pattern of the flow-volume loop did not support the diagnosis of extrathoracic, upper airway causes of obstruction. Methacholine challenge had not been done because of severely reduced $\mathrm{FEV}_{1}$ and the test's poor specificity in differentiating causes of obstruction. An echocardiogram showed an elevated right ventricular systolic pressure of $40 \mathrm{~mm} \mathrm{Hg}$ (normal $15-30 \mathrm{~mm} \mathrm{Hg}$ ). High-resolution computed tomography (CT) scans showed a few areas of triangular subpleural opacities, linear atelectasis, mild septal thickening and evidence of small airways disease.

\section{What other diagnosis or diagnoses should be considered?}

a. Cystic fibrosis

b. Alpha-antitrypsin deficiency

c. Allergic bronchopulmonary aspergillosis

d. Nonadherence to therapy

e. All of the above

Given the patient's difficult-to-control disease and low diffusion capacity, we entertained other causes of airways obstruction besides severe asthma (e). Workup for allergic bronchopulmonary aspergillosis, cystic fibrosis and $\alpha_{1}$-antitrypsin deficiency ruled out these diagnoses. We also ruled out silent aspiration of food and liquid. As for the possibility
Competing interests: None declared.

This article was peer reviewed.

Correspondence to:

Vivek Dhawan,

vdhawan@ualberta.ca

CMAJ 2013. DOI:10.1503 /cmaj.110952 
of nonadherence to therapy, the patient's support worker assured us that the patient had been taking her medications, and her inhaler technique was observed to be adequate.

\section{What additional information will be helpful to establish the diagnosis?}
a. Bronchoscopy
b. Obtain a detailed birth and neonatal history
c. Lung biopsy
d. Repeat pulmonary function testing
e. Have the patient keep a symptom diary

A birth and neonatal history will help to determine the diagnosis (b). No such history had been obtained during our patient's previous investigations. Her support worker did not have this information. We contacted the patient's mother, who informed us that the patient had had a complicated neonatal period requiring a prolonged hospital stay. We reviewed the patient's neonatal medical records, which indicated that she was born at 23 weeks' gestation and her birth weight

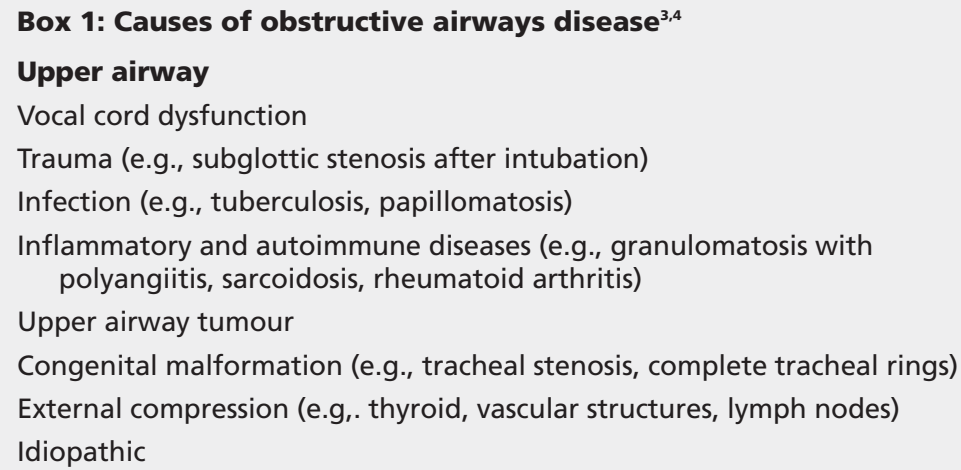

was $0.53 \mathrm{~kg}$. She required mechanical ventilation for 2 months and further oxygen therapy for 5 months. Her stay was complicated, with multiple episodes of pneumonia; no pneumothorax was noted in her history. She had feeding difficulties and needed to be fed through a nasogastric tube for a prolonged period. She also had patent ductus arteriosus that was ligated. There was no history of oligohydramnios. The patient was then lost to follow-up until recently, when she started seeking medical help because of shortness of breath and recurrent "asthma" exacerbations.

With this additional birth and neonatal history, we diagnosed bronchopulmonary dysplasia (BPD). Over the course of a week in hospital, the patient's condition improved and she was discharged. She is taking an inhaled corticosteroid and long-acting $\beta$-agonist, with shortacting bronchodilators as rescue medication. She has stopped smoking and was advised to lose weight. She will not likely be a good candidate for lung transplant because of her developmental disability and poor social support.

\section{Discussion}

Although most patients with obstructive airways disease have asthma or chronic obstructive pulmonary disease (COPD), less common pulmonary conditions can present in a similar manner $^{1,2}$ (Box 1,4). Uncommon features such as atypical disease progression or a poor response to standard treatment should encourage clinicians to seek other causes of airflow limitation by taking a thorough history and requesting other tests as indicated. A diagnosis of difficult-to-treat asthma should be considered, and factors such as medication adherence, inhaler technique, exposure to allergens, smoking status and comorbidities such as sinusitis and gastroesophageal reflux should be explored. If the more common diagnoses of asthma and COPD are ruled out, rarer causes of obstruction should be considered., ${ }^{1,2}$

Obtaining a complete birth and neonatal history should be standard practice in all cases of obstructive lung disease. In the case we have described, such a history proved be a vital part of our investigations and helped with the diagnosis.

\section{Bronchopulmonary dysplasia}

Bronchopulmonary dysplasia (BPD), a chronic lung disease affecting premature neonates, is defined as continued dependence on supplemental oxygen for more than 28 days after birth. ${ }^{5}$ It is mainly seen in neonates born before 30 weeks' gestation with a birth weight of less than $1500 \mathrm{~g}$.

The disease is classified as mild, moderate or severe depending on the duration and amount of 
oxygen required near term. It is also classified as old or new depending on the use of surfactant treatment and the timing and extent of lung injury due to mechanical ventilation. Detailed diagnostic criteria and severity grades of this condition have been developed.

About 60000 premature infants are born annually in the United States, $20 \%$ of whom will have BPD. ${ }^{6}$ The epidemiologic features among adults with the disease are less clear. Up to $25 \%$ $71 \%$ of those with BPD who survive into adulthood will continue to have respiratory symptoms at that time. ${ }^{7}$

\section{Pathogenesis}

Old BPD occurs in slightly premature neonates not given surfactant who are exposed to aggressive mechanical ventilation and supplemental oxygen treatment. This management leads to intense inflammation and massive parenchymal disruption in surfactant-deficient lungs. The incidence of old BPD has declined substantially because of advances in ventilation modalities and use of steroids and surfactant in premature neonates. ${ }^{6}$

In contrast, new BPD, considered a developmental disorder, is a milder form of the disease that affects very premature neonates given surfactant. The underdeveloped lungs of these neonates are exposed to various insults, which leads to impaired alveolization and vascularization. The result is simplification of lung architecture, with fewer and larger alveoli. Airways are relatively spared, and inflammation is milder than in the old form of the disease. ${ }^{6}$

Recently, genetic research in BPD suggested that abnormalities in the genes involved in angiogenesis, inflammation and immune factors such as surfactant, tumour necrosis factor $\alpha$ and antioxidants may play a role in predisposing patients to BPD. ${ }^{8}$

The effects of prolonged intubation and oxygen therapy are not only limited to the small airways and alveolar space but can also cause trauma to the upper and central airways. The trauma may be the result of intubation or frequent suctioning. This can lead to tracheobronchomalacia, which can further worsen the obstructive physiology of BPD.

\section{Sequelae in adulthood}

Although BPD during infancy has been studied extensively, only a few studies have looked at the long-term sequelae of BPD. It is clear, however, that adults with BPD have an increased incidence of respiratory symptoms, including cough, wheezing and asthma. Northway and colleagues ${ }^{9}$ studied pulmonary outcomes in 26 young adults with BPD and found that $25 \%$ of the patients had re- current respiratory difficulties, including wheezing, episodes of pneumonia and limited exercise capacity.

\section{Lung function}

Results of pulmonary function testing of patients with BPD will show persistent airway obstruction, airway hyperresponsiveness and hyperinflation of the lungs. ${ }^{9-11}$ In a study of the effect of preterm birth on pulmonary function at age 7 years, preterm children with BPD had significantly reduced $\mathrm{FVC}$ and $\mathrm{FEV}_{1}$ compared with healthy children who were born at term. ${ }^{12}$ These abnormalities may persist into adulthood. ${ }^{9}$ Not only is lung function worse in patients with BPD, but the rate of decline is faster than normal. In a study involving 147 people who had a birth weight of less than $1500 \mathrm{~g}$ and who underwent pulmonary function testing at a mean age of 18.9 years, those who had BPD in the neonatal period had clinically significant reductions in airflow and deterioration in pulmonary function since the previous evaluation at 8 years of age. ${ }^{13}$ Birth weight ${ }^{13}$ and results of pulmonary function testing at 2 years of age ${ }^{14}$ appear to be reasonable predictors of long-term pulmonary outcome, with low birth weight and abnormal test results indicating a poorer prognosis.

Contrary to the above-mentioned findings of abnormal lung function persisting into adulthood in people with BPD, Narang and colleaegues ${ }^{15}$ have proposed that lung function can be regained over time in patients with BPD. However, they acknowledged that limitations in the study may have contributed to this finding. ${ }^{15}$

\section{Pulmonary hypertension}

Another feature of BPD is the development of pulmonary hypertension. ${ }^{16}$ Pulmonary hypertension develops early during infancy and is a predictor of poor survival. Bronchopulmonary dysplasia results in a reduced pulmonary capillary bed and increased vascular tone, which leads to increased pulmonary arterial pressures. Small airways obstruction may also contribute to pulmonary hypertension in these patients. Those with BPD and pulmonary hypertension who survive infancy usually show improvement in pulmonary pressures over time. ${ }^{16}$

\section{Radiographic findings}

Persistent radiographic changes are observed in patients with BPD. ${ }^{11}$ In adults, the most common findings on high-resolution CT scans include linear and triangular subpleural opacities, emphysema and thickening of the bronchial wall. ${ }^{11}$ Other findings include mosaic perfusion and air trapping. These findings can exist even in the 
absence of overt clinical symptoms. The duration of oxygen therapy during infancy seems to be an important predictor of structural abnormalities on high-resolution CT scans seen later in life. ${ }^{11}$

\section{Nonpulmonary changes}

There are nonpulmonary long-term consequences of BPD, especially in the areas of cognitive and motor development. A recent systematic review identified 2 studies that reported developmental delay and learning difficulties in adults with BPD compared with those born prematurely without BPD. ${ }^{17}$ Although not seen consistently, anthropometric data from patients with BPD implies growth failure in adulthood. ${ }^{17}$

\section{Association with asthma}

The underlying pathophysiology of asthma differs from that of BPD. However, these conditions may present in a similar manner clinically. In the study by Northway and colleagues, ${ }^{9}$ symptoms, signs and findings on pulmonary function testing similar to those of asthma were reported in up to $50 \%$ of the participants with a history of BPD in infancy. The incidence of airway hyperreactivity is higher among children with a history of BPD than among those without such a history. ${ }^{6}$ Several other studies have noted an increased history of asthma among young adults with BPD, but the reported rates have varied.? Lack of standardization of the diagnostic criteria for asthma in these studies could explain the observed variation. It has also been suggested that, because of a substantial overlap between asthma and BPD symptoms, some children are imprecisely labelled as having asthma. ${ }^{6.14}$

\section{Diagnosis}

Because BPD shares symptoms with other obstructive airways diseases, it can be clinically challenging to diagnose it. Furthermore, as with other childhood-onset diseases, BPD may be forgotten and not included in the differential diagnosis of obstructive airways disease in an adult patient. Several clues, however, should raise suspicion of BPD. The most important is the birth and neonatal history. A history of low birth weight, prematurity, prolonged use of supplemental oxygen and the need for mechanical ventilation upon birth supports the inclusion of BPD in the differential diagnosis. Other clues include unusual severity of the obstructive airways disease for the patient's age and a lack of smoking history. Although commonly associated with asthma, the prevalence of atopy or a family history of asthma was not increased among children with BPD compared with controls in the study by Northway and colleagues. ${ }^{9}$
On investigation, the airflow limitation in BPD may be only partially reversible after use of a bronchodilator. ${ }^{18}$ There are also differences between findings of asthma and BPD on highresolution CT scans. Scattered parenchymal fibrosis and distortion of the lung architecture, common findings in BPD, are rarely seen in asthma. The role of sputum analysis is established in asthma, but its role in BPD is unclear. In a study involving 31 children with BPD, the levels of exhaled nitric oxide, a marker of eosinophilic inflammation, were significantly lower in those with BPD than in age-matched children with asthma. ${ }^{19}$ In complex cases with added elements of cigarette smoking and drug abuse, such as the case with our patient, it is particularly challenging to diagnose BPD.

The failure to respond to standard treatments of obstructive airways diseases is another important clue in identifying BPD. The response to inhaled or systemic corticosteroid therapy in patients with BPD is less consistent than in patients with asthma. ${ }^{6,20}$

\section{Treatment}

Although there has been much interest in the treatment of BPD in premature neonates around the time of birth, the optimal therapy in adults with BPD is not known. Bronchopulmonary dysplasia is considered to be a developmental disorder, and our current understanding of its pathophysiology does not support the use of systemic corticosteroid therapy. In the absence of evidencebased studies, such treatment may be harmful. Because there are no forthcoming guidelines for the management of BPD in adults, we suggest that long-term steroid therapy be avoided and the cause of acute exacerbations be investigated and treated accordingly. Furthermore, the role of inhaled corticosteroid therapy is unclear. In a study involving 15 children of low birth weight, regular use of inhaled corticosteroids did not alleviate symptoms or improve lung function..$^{20}$

The use of intravenous immunoglobulin has been reported to be of benefit in infants with recurrent infection; again, there are no data to support this treatment in adults. ${ }^{21}$ Bronchopulmonary dysplasia is an indication for lung transplant in infants, and there is a case report of lung transplant in a 19-year-old patient with BPD. ${ }^{22}$ With improvements in the understanding of mechanisms of lung repair and inflammation in BPD, new treatment strategies are being explored. Enzyme, gene, cytokine, antioxidant and antiprotease therapies as well as stem cell treatment are being developed in animal models of lung injury. ${ }^{23}$

Every effort should be made to minimize further insult to the lungs. These include smoking 
cessation and regular vaccinations. In our patient, we believe that the cause of her frequent exacerbations was cigarette smoking. The patient was duly counselled and has quit smoking.

Although it might appear that there is no therapeutic advantage to diagnosing BPD, it is nevertheless important to patient care. Making the right diagnosis will not only discourage physicians from pursuing more aggressive investigations, it may also minimize potential adverse effects of treatments such as long-term systemic steroid therapy that may have little added clinical benefit.

\section{Conclusion}

With advances in the care of premature neonates, more children with BPD will reach adulthood and will present with symptoms shared by more common obstructive airways diseases such as asthma and COPD. The case we have described highlights the importance of taking a thorough medical history, including a birth and neonatal history. Furthermore, it also illustrates that childhoodonset diseases can have sequelae in adulthood and should be included in the differential diagnosis in adults presenting with chronic dyspnea.

\section{References}

1. Global strategy for asthma management and prevention. Global Initiative for Asthma; 2012. Available: www.ginasthma.org /documents/4 (accessed 2013 Mar. 20).

2. Global strategy for the diagnosis, management and prevention of chronic obstructive pulmonary disease (updated 2013). Global Initiative for Chronic Obstructive Lung Disease; 2011. Available: www.goldcopd.org/uploads/users/files/GOLD_Report 2013_Feb20.pdf (accessed 2013 Mar. 20).

3. Mason RJ, Nadel JA, Murray JF. Murray and Nadel's textbook of respiratory medicine. 5th ed. Philadelphia (PA): Elsevier Saunders; 2010;1066-79.

4. Gaissert HA, Burns J. The compromised airway: tumors, strictures, and tracheomalacia. Surg Clin North Am 2010;90:1065-89.

5. Jobe AH, Bancalari E. Bronchopulmonary dysplasia. Am J Respir Crit Care Med 2001;163:1723-9.

6. Baraldi E, Filippone M. Chronic lung disease after premature birth. N Engl J Med 2007;357:1946-55.

7. Narang I. Review series: What goes around, comes around: Childhood influences on later lung health? Long-term follow-up of infants with lung disease of prematurity. Chron Respir Dis 2010;7:259-69.

8. Lavoie P, Dube M. Genetics of bronchopulmonary dysplasia in the age of genomics. Curr Opin Pediatr 2010;22:134-8.

9. Northway WH, Moss RB, Carlisle KB, et al. Late pulmonary sequelae of bronchopulmonary dysplasia. N Engl J Med 1990; 323:1793-9.

10. Wong P, Lees A, Louw J, et al. Emphysema in young adult survivors of moderate-to-severe bronchopulmonary dysplasia. Eur Respir J 2008;32:321-8.

11. Aukland S, Rosendahl K, Owens C, Neonatal bronchopulmonary dysplasia predicts abnormal pulmonary HRCT scans in longterm survivors of extreme preterm birth. Thorax 2009;64:405-10.

12. Gross SJ, Iannuzzi DM, Kveselis DA, et al. Effect of preterm birth on pulmonary function at school age: a prospective controlled study. J Pediatr 1998;133:188-92.

13. Doyle LW, Faber B, Callanan C, et al. Bronchopulmonary dysplasia in very low birth weight subjects and lung function in late adolescence. Pediatrics 2006;118:108-13.

14. Filippone M, Bonetto G, Cherubin E, et al. Childhood course of lung function in survivors of bronchopulmonary dysplasia. JAMA 2009;302:1418-20.

15. Narang I, Rosenthal M, Cremonesini D, et al. Longitudinal evaluation of airway function 21 years after preterm birth. Am J Respir Crit Care Med 2008;178:74-80.

16. Khemani E, McElhinney D, Rhein L, et al. Pulmonary artery hypertension in formerly premature infants with bronchopulmonary dysplasia: clinical features and outcomes in the surfactant era. Pediatrics 2007;120:1260-9.

17. A Gough, D Spence, M Linden, HL et al. General and respiratory health outcomes in adult survivors of bronchopulmonary dysplasia: a systematic review. Chest 2012;141:1554-67.

18. Pelkonen AS, Hakulinen AL, Turpeinen M. Bronchial lability and responsiveness in school children born very preterm. Am J Respir Crit Care Med 1997;156:1178-84.

19. Baraldi E, Bonetto G, Zacchello F, et al. Low exhaled nitric oxide in school-age children with bronchopulmonary dysplasia and airflow limitation. Am J Respir Crit Care Med 2005;171:68-72.

20. Chan KN, Silverman M. Increased airway responsiveness in children of low birth weight at school age: effect of topical corticosteroids. Arch Dis Child 1993;69:120-4.

21. Wheeler W, Kurachek S, McNamara J, et al. Consequences of hypogammaglobulinemia and steroid therapy in severe bronchopulmonary dysplasia. Pediatr Pulmonol 1996;22:96-100.

22. Starnes VA, Barr ML, Schenkel FA, et al. Experience with living-donor lobar transplantation for indications other than cystic fibrosis. J Thorac Cardiovasc Surg 1997;114:921-2.

23. Alphonse RS, Thébaud B. Growth factors, stem cells and bronchopulmonary dysplasia. Neonatology 2011;99:326-37.

Affiliations: Department of Medicine, Division of Pulmonary Medicine, University of Alberta, Edmonton, Alta.

Contributors: Both authors contributed equally to the conception of the article, and the drafting and revising of the manuscript for important intellectual content, Both authors approved the final version submitted for publication. 